\title{
Assessing olive oil peroxide value by NIRS, and on reference methods
}

José A. Cayuela, Instituto de la Grasa, CSIC. University Campus Pablo de Olavide, Ed. 46., ctra. Utrera, Km. 1, 41013 Seville, Spain. Email: jacayuela@ig.csic.es

\section{Introduction}

Chemical analysis methods have its own accuracy and precision. These depends on the sample preparation procedure, the degree of instrumentation staking on the method and its characteristics. We will not refer here to techniques based on spectral characteristics which do not require reference analytical values, such as PLS-DA. When analytical techniques base on calibrations involving any correlation, whether simple or multivariate, their accuracy depends directly on the accuracy and precision of the reference method. This is the case with NIR or Vis/NIR, using the correlation between certain wavelengths and the reference values from a well recognized method of chemical analysis. Therefore, it will be impossible a priori improving the reference method accuracy with a NIR model. Unless it is assumed the reference accuracy is not best, thus using some correction.

This topic is not new, and it will surely have been the concern of many people involved in developing innovative analytical methods, as reflected in the literature ${ }^{1}$. However, I think in the NIR spectroscopy this problem is especially significant. NIR spectrum contains information on the set of physical and chemical properties of matter. Thus, specific chemical features often correlate better with this integrated information than with isolated markers. The issue that motivates the present paper is in certain cases NIR spectroscopy may provide physicochemical information enough to set excellent quantitative models. These models, however, sometimes may be difficult to achieve. One reason can be the reference method lacks characteristics of excellence in accuracy and precision. We should highlight if we want to get a good NIR technique, we should be aware of the interest to improve reference method, when suitable. Or put another way, it may be advisable spending effort extra in reference analysis, if it has as compensation achieving a new analytical technique which will soon compensate these costs. When working to set up NIR calibrations for predicting the peroxide value in olive oils, we have been thinking about this idea. I will try to provide a clear picture of the problem I am referring to, distracting for a moment the attention of the reader by presenting a different example.

Keywords: olive oil; reference methods; peroxide value.

\section{Wine sensory attributes}

In some food sensory analysis assessing certain attributes is according to continuous scales. However, it is normal for the taster ratings to show a tendency to repetitive unit values. It occurs in particular with low intensity attributes or defects. The Fig. 1 shows this trend, referred to the 
astringency of different white wines. The fact highlights a relative lack of accuracy of the method. At the same time, it reveals the possibility of a NIR assessment with higher accuracy ${ }^{2}$. In this sense, Andersson et al. ${ }^{3}$ reported a method for finding out when NIR is better than a reference analysis. This method needs circulating and analysing $\mathrm{N}$ samples using the same analytical method at different locations with different operators. However, this does not solve the problem of achieving a good and robust model.

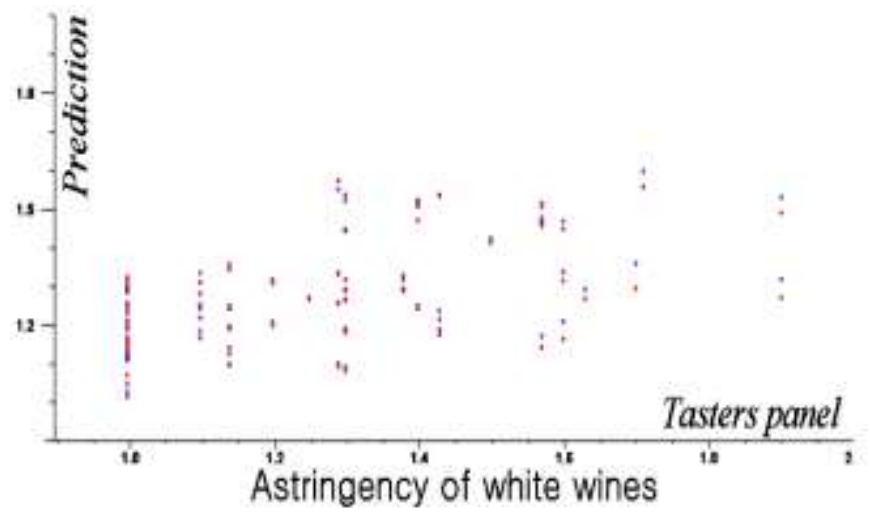

Figure 1. Vis/NIR prediction of white wine astringency.

\section{Olive oil peroxide value}

Back to the peroxide value (PV), this is an important olive oil quality parameter signalling the degree of oxidative alteration of the product. The analysis method is included in Annex III of the EEC Regulation 2568/91 on the characteristics of olive oils and olive-pomace oils, and on their methods of analysis. It is as well fixed as standard method by the International Olive Council (IOC), updated recently. This method defines the PV as the peroxides in the sample, expressed as milliequivalents of active oxygen per $\mathrm{kg}$ of fat, which causes the oxidation of the potassium iodide under the described working conditions. The analytical principle consists in the titration with sodium thiosulphate of the liberated iodine when treating with the potassium iodide solution the oil sample, previously dissolved in acetic acid and chloroform. The procedure, in brief, consists of weighing in a $250 \mathrm{ml}$ flasked Erlenmeyer flask 1.8 to $2.2 \mathrm{~g}$ of previously filtered olive oil. Add $25 \mathrm{~mL}$ of acetic / chloroform in $3: 2$ ratio, adding $1 \mathrm{~mL}$ of saturated potassium iodide, prepared at the same time. Stir gently and leave for 5 minutes in the dark, whereupon $75 \mathrm{~mL}$ of bidistilled $\mathrm{H}_{2} \mathrm{O}$ are added and the mixture is stirred vigorously. The titration is performed with $0.002 \mathrm{~N}$ thiosulfate to straw yellow color. 20 drops of starch solution are added, whereby the sample is darkened, which is continued titrating until it turns white. I would like highlight this last step is especially delicate, because the turning is progressive. Therefore, to get good accuracy and precision in the determination, the analyst must be aware and careful with the method.

The method precision has been determined from results of collaborative tests by the IOC, pointing out reproducibility $\left(\mathrm{CV}_{\mathrm{r}}\right)$ and repeatability $\left(\mathrm{CV}_{\mathrm{R}}\right)$ coefficients of variation $7.1 \%$ and $1.9 \%$ for extra virgin olive oils, the same statistics being $13.8 \%$ and $3.4 \%$ for normal olive oils.

Measuring PV of vegetable oils by NIR spectroscopy has been reported several times in the literature, although may highlight two works for their specificity regarding olive oil. Mailer ${ }^{4}$ reported a regression coefficient of cross validation 0.85 , although no external validations were performed. Armenta et al. ${ }^{5}$ reported an external validation with $\mathrm{r}^{2} 0.66$ using a calibration for predicting olive oil PV.

We have carried out several NIR calibration tests in our lab for predicting the PV. One of the first assays was made with 369 samples of olive oils diverse, using a NIR (1100-2300 nm) spectrometer 
(ASD, Boulder) and transflectance mode acquisition. This last consists on a stainless steel probe with threaded interchangeable optical paths. The NIR radiation coming from the optical fiber goes through the liquid sample, reflects in the polished stainless steel surface of the optical path's cylinder. Then, it back through the liquid sample, heading to the spectrometer detector. The whole spectrum was registered, each spectral variable matching to a $2 \mathrm{~nm}$ interval. Selecting the spectral variables involved in the models was made by consecutive cycles removing those which the contribution were closer to zero. Variable selection ended in the last cycle that improved the statistical model $\mathrm{R}_{\mathrm{CV}}^{2}$. The methodology used was similar for all the tests forward.

The above referred test provided the calibration $\left(C_{l}\right)$ depicted in Fig. 2. The repeatability coefficient of variation of the reference method $\left(C_{R}\right)$ was about 3.7\%. The calibration and cross validation $R^{2}$ and $R_{C V}^{2}$ coefficients, once six reference values suspected of analytical error have been discarded, were 0.64 and 0.58 , what is not enough to provide good measures. A external validation, shown in Fig. $2\left(V_{1}\right)$, was carried out by predicting on 123 new samples confirming this forecast, with RPD as low as 1.33. For those unfamiliar with the interpretation of this statistic, it is assumed that from RPD values close to 3 , the models would be suitable for routine analysis.
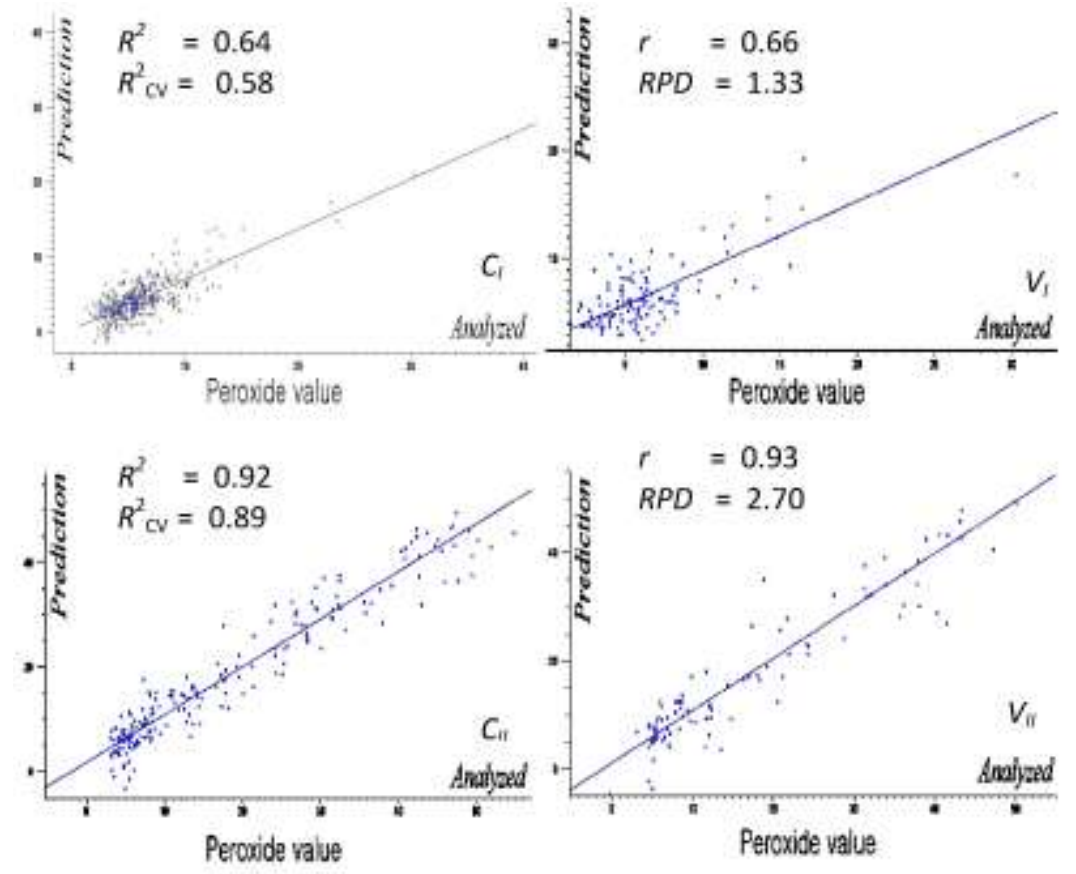

Figure 2. NIR tests of olive oil peroxide value. Calibrations and validations.

Some time later, we try improving the PV calibrations by using three replicates of the reference analysis for each sample, instead two replicates which is most usual. The $\mathrm{CV}_{R}$ was about $4 \%$.

According to this statistic, no improvement over the determinations performed with two replicates was reached. The model (Fig. 2, $C_{I I}$ ), using 192 diverse olive oils, showed $R^{2}$ of 0.92 , and $R_{C V}^{2}$ of 0.89 . A prediction exercise (Fig. 2, $V_{I I}$ ) was performed using this model on 101 new olive oils samples from different origins, and this external validation provided statistics $r$ of 0.93 and RPD of 2.70. Thus, the outcomes in practice were better. I wonder to what extent the modification of the reference method was involved in this, despite the $\mathrm{CV}_{R}$ showed no improvement. 
We assumed the task of evaluating the contribution of visible wavelengths together with NIR on calibrations for the PV measurement of olive oil. For this purpose we used 321 olive oil samples with great diversity on their origins and types, that could be interesting for good robustness. However, their whole traceability data were available only for a part of the samples. The equipment used is shown in Fig. 3.

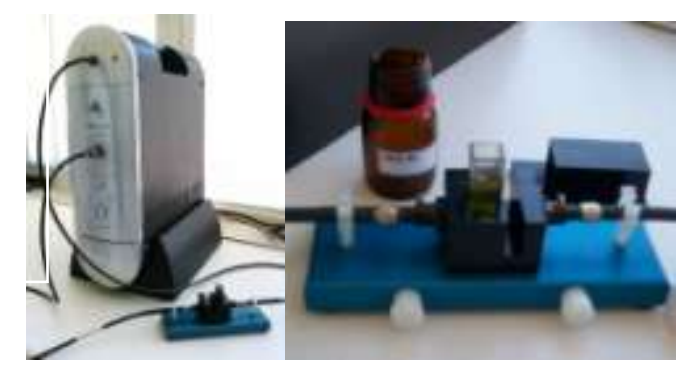

Figure 3. Labspec (ASD Inc., Boulder) spectrometer and cuvette holder (Ocean Optics Inc.).

The dispersion plot of the calibration built is shown in Fig. $4\left(C_{\text {III }}\right)$. A large number of visible variables seemed to participate (data not shown), although them probably integrates some noise also. External validation was carried out by predicting on 107 new diverse olive oils samples, and this exercise (Fig. 4, $V_{I I I}$ ) provided statistics $r$ of 0.90 and RPD of 2.11. This indicates the model prediction accuracy is not enough for routine analysis.
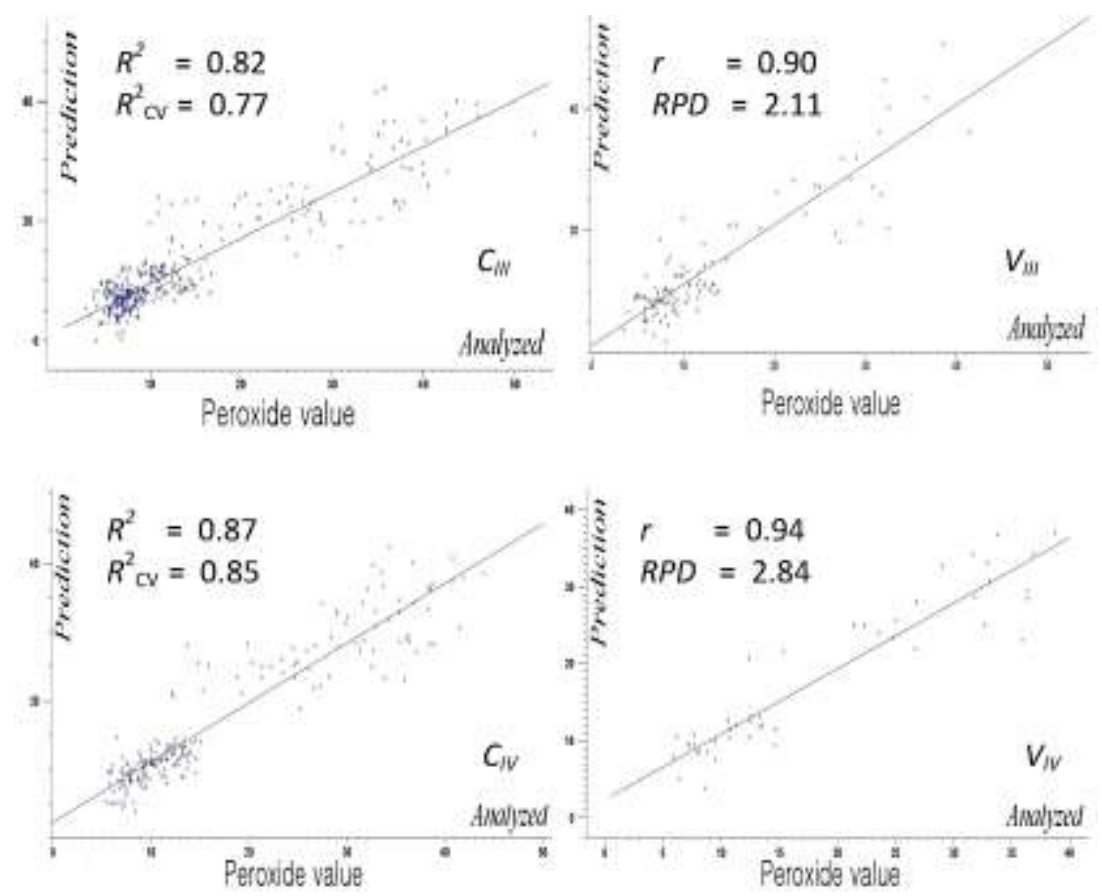

Figure 4. Vis/NIR tests of olive oil peroxide value. Calibrations and validations. 
We try again, by using a set of 199 olive oils. This Vis/NIR calibration (Fig. 4, $C_{I V}$ ) assay gave slightly better outcomes than that above, with $R^{2}$ of 0.87 , and $R^{2}{ }_{c v}$ of 0.85 . In this calibration participated approximately the same spectral variables as in $C_{\text {III. }}$. It was checked in this calibration that eliminating the visible wavelengths worsened both its adjustment and its operation. However, I must say I think the actual contribution of the visible spectrum should be further clarify. The external validation (Fig. $4, V_{I V}$ ), carried out on 46 samples independent from the calibration set, provided RPD of 2.84 .

Considering the above, I believe it can be said that in the future the NIR or Vis/NIR techniques may be recognized as an official and advantageous method for the analysis of olive oil peroxide value, after the necessary steps!.

\section{References}

1. P. Paash, "Myth: A partial least squares calibration model can never be more precise than the reference method...", NIR News, 25(3), 20-23 (2014).

2. J. A. Cayuela, B. Puertas, E. Cantos-Villar, "Assessing wine sensory attributes using Vis/NIR", Europ. Food. Res. Technol. , doi:10.1007/s00217-016-2807-9 (2016).

3. M. Andersson, L. Nørgaard, "A procedure to determine when NIR is better than its reference method", in Near Infrared Spectroscopy: Proceedings of the 16th International Conference, La Grande-Motte - France, 2-7 June (2013).

4. R. J. Mailer, "Rapid evaluation of olive oil quality by NIR reflectance spectroscopy", JAOCS, 81, 823-827 (2004).

5. S. Armenta, S. Garrigues, M. De la Guardia, "Determination of edible oil parameters by near infrared spectrometry", Anal. Chim. Acta 596, 330-337 (2007). 